\title{
The risk factors of vertebral refracture after kyphoplasty in patients with osteoporotic vertebral compression fractures: a study protocol for a prospective cohort study
}

Lei Feng $^{1+}$, Chun Feng ${ }^{2+}$, Jie Chen ${ }^{1}, Y u W^{1}$ and Jin-Ming Shen ${ }^{1 *}$ (D

\begin{abstract}
Background: Percutaneous kyphoplasty (PKP) is the first-line treatment for osteoporotic vertebral compression fractures (OVCFs) that can immediately relieve pain and allow the quick recovery of lost mobility. However, some studies reported that after PKP, the incidence of vertebral refracture, particularly adjacent vertebral fracture (AVF), was high. Our previous meta-analysis suggested that the risks for vertebral refracture and AVF did not increase after percutaneous vertebral augmentation in OVCF patients. Despite the negative results of our meta-analysis, there is still significant evidence regarding the relationship between kyphoplasty and AVF, so a new prospective cohort study is warranted. In addition, in our previous retrospective study, we found that advanced age, female sex and low oestradiol $\left(E_{2}\right)$ concentrations might be related to the occurrence of postoperative vertebral refracture after PKP. To sufficiently evaluate the probable factors involved in the occurrence of postoperative vertebral refracture, we designed this prospective study.

Methods: This is a prospective cohort study of patients admitted for PKP to treat painful OVCFs. The baseline data, including demographic information, lifestyle, bone metabolic status, sex hormone and sex hormone-binding globulin (SHBG) levels, and clinical characteristics will be collected the time of enrolment. Surgical features of PKP will be recorded on the operation day. Lifestyle, bone metabolic status, sex hormone levels, and SHBG levels will be assessed during the follow-up period at $1 \mathrm{~m}, 3 \mathrm{~m}, 12 \mathrm{~m}$, and $24 \mathrm{~m}$ postoperatively. Patients suffering from acutely aggravated back pain will be referred to an orthopaedist, and refractured vertebrae will be confirmed by magnetic resonance imaging and computed tomography. The primary outcome will be the incidence of vertebral refracture. Multivariate analyses will be carried out to evaluate the variables that are independently correlated with vertebral refracture.
\end{abstract}

Discussion: To evaluate the risk of postoperative refracture preoperatively and to identify the surgical points related to postoperative refracture, this study will explore the risk factors related to vertebral refracture after PKP. The results may provide new information about defining OVCF patients suitable for PKP treatment.

Trial registration: ChiCTR-ROC-17011562. Registered on July 4th, 2017.

Keywords: Osteoporotic vertebral compression fracture (OVCF), Kyphoplasty, Refracture, Bone mineral density (BMD)

\footnotetext{
* Correspondence: shenjinmg@gmail.com

†Lei Feng and Chun Feng contributed equally to this work.

'Department of Orthopaedics, The First Affiliated Hospital of Zhejiang

Chinese Medicine University, 9 Ninth Avenue, Hangzhou Economic and

Technological Development Zone, Zhejiang 310018, Hangzhou, China

Full list of author information is available at the end of the article
}

(c) The Author(s). 2018 Open Access This article is distributed under the terms of the Creative Commons Attribution 4.0 International License (http://creativecommons.org/licenses/by/4.0/), which permits unrestricted use, distribution, and

reproduction in any medium, provided you give appropriate credit to the original author(s) and the source, provide a link to the Creative Commons license, and indicate if changes were made. The Creative Commons Public Domain Dedication waiver (http://creativecommons.org/publicdomain/zero/1.0/) applies to the data made available in this article, unless otherwise stated. 


\section{Background}

Every year, there are approximately $1,110,000$ people in China [1] and 700,000 people in the United States [2] who suffer from osteoporotic vertebral compression fractures (OVCFs). Therefore, the treatment of these patients is an important topic to improve patients' quality of life and reduce the burden on society. The first-line therapy for OVCF patients is conservative treatment, including medication, orthotic bracing, and physical therapy. In recent years, percutaneous kyphoplasty (PKP) has become more prevalent worldwide because it can rapidly relieve pain and dramatically improve patients' quality of life.

A series of studies have compared the therapeutic effects between conservative treatment and percutaneous vertebral augmentation. In 2009, a multicenter randomized controlled trial (RCT) compared 68 OVCF patients who underwent vertebroplasties and 63 patients who underwent simulated procedures (control group) and found that improvements in pain and pain-related disability were similar between the two groups [3]. However, in 2011, an RCT performed in Belgium that compared 149 cases treated with kyphoplasty and 151 cases treated with conservative therapy found that kyphoplasty rapidly reduced pain and improved function, disability, and quality of life, without increasing the risk of additional vertebral fractures [4]. In 2013, a multicenter RCT performed in Europe compared kyphoplasty and conservative therapy for the treatment of acute painful vertebral fractures and found that kyphoplasty achieved pain relief and functional recovery and rapidly improved patients' quality of life [5]. In 2016, a multicenter double-blind RCT performed in Australia indicated that in patients with acute OVCF, vertebroplasty was superior to placebo for pain relief [6]. A recent meta-analysis involving ten RCTs indicated that compared with conservative therapy, vertebroplasty/ kyphoplasty was associated with greater pain relief, significant improvement in daily function, and higher quality of life [7]. The majority of studies support the finding that vertebroplasty/kyphoplasty is beneficial for acute OVCF.

However, some reports suggested that the risk of new vertebral fractures might be elevated after percutaneous vertebral augmentation. More than ten years ago, a retrospective analysis performed in the USA suggested that after vertebroplasty the risk of new vertebral fractures increased, particularly adjacent level fractures, and found that they occurred earlier than non-adjacent level fractures [8]. However, the subsequent studies reported inconsistent results and did not support the correlation between PKP and vertebral refracture [9]. There have been several RCT studies investigating the risk of new vertebral fractures after percutaneous vertebral augmentation in patients of OVCF all over the world, including in China [10-13], Iran [14], Australia [15-17], the
Netherlands [18, 19], Spain [20], and Denmark [21]. We reviewed these studies and performed a meta-analysis, which suggested no increase in the risk of new vertebral fracture or adjacent vertebral fracture [22].

If the high incidence of vertebral refracture is not associated with PKP, it is possible that underlying osteoporosis is the leading factor that results in the onset of vertebral fracture rather than percutaneous vertebral augmentation. Advanced age and decreased bone mineral density (BMD) scores were found to be correlated with the risk of AVF following PVP [23]. We carried out a retrospective study and found that advanced age, female sex and low oestradiol $\left(\mathrm{E}_{2}\right)$ concentrations might be risk factors associated with postoperative vertebral refracture after kyphoplasty [24].

To more clearly and sufficiently evaluate the probable factors involved in the occurrence of postoperative vertebral refracture, we designed this prospective study to explore these risk factors. According to published reports and our previous study, we focused on the following aspects. 1) General patient characteristics may be important because advanced age is generally recognized as a risk factor for new vertebral fracture after vertebral augmentation, but it is unclear whether there should be an age limit for PKP treatment and what the cutoff age is [23-25]. 2) Bone metabolic status, which is reflected by $\mathrm{BMD}$, serum bone metabolic markers, and the effect of anti-osteoporotic treatments, may be one of the dominant risk factors for refracture [26, 27]. 3) Sex hormone levels may be important, because numerous studies reported the association between sex hormone binding globulin (SHBG) and new or worsening vertebral fracture, although no association with $\mathrm{E}_{2}$ was found, indicating a role of SHBG in the pathogenesis of postoperative vertebral refracture [28-30]. Our previous study found that low $\mathrm{E}_{2}$ concentration might be associated with vertebral refracture after kyphoplasty [24]. 4) Some surgical factors, including balloon volume, cement volume, recovery of vertebral height, and cement leakage may be correlated with the incidence of vertebral fracture after kyphoplasty [26, 31]. 5) Lifestyle factors may also be involved. A study in the United States found that male smokers had a significant risk of low volumetric BMD and vertebral fractures [32]. Tobacco consumption may also be an independent predictor of non-vertebral fracture [33]. Although previous studies showed that these factors may be involved in fracture recurrence, more studies are required to further explore the role of these different factors. This prospective cohort study is designed to investigate the factors involved in the occurrence of postoperative refracture, which may provide some useful information for defining the target population for kyphoplasty and improving surgical techniques. If this study can be carried out successfully, it may be 
helpful for reducing surgical complications such as the occurrence of new vertebral fractures.

\section{Primary objectives}

- To explore the possible factors involved in the occurrence of postoperative refracture, such as general characteristics, bone metabolic status, antiosteoporotic treatment, sex hormone and SHBG levels, and the PKP procedures.

- To clarify and define the patients with OVCF suitable for PKP treatment with a low risk of postoperative refracture.

\section{Methods}

\section{Study design}

The present study is a prospective cohort study. Participant recruitment for the study will begin in Apr 2018 and continue until Mar 2019. As shown in Fig. 1, patients with OVCF who are admitted to the First Affiliated Hospital of Zhejiang Chinese Medicine University will be assessed by an orthopaedist to ensure that they are eligible for the study and will sign an informed consent form and be enrolled in this study. All the candidate patients will be recorded in a log that contains the demographic data and the reasons for non-participation in the study. The study protocol has been approved by the Ethics Committee of the First Affiliated Hospital of Zhejiang Chinese Medicine University.

\section{Study setting}

This study will be carried out at the First Affiliated Hospital of Zhejiang Chinese Medicine University, which is a tertiary care centre that integrates medical treatment, medical education, scientific research, rehabilitation and healthcare. The hospital occupies 6.13 ha of land with 102,000 square metres of floor space for medical service and has 1500 inpatient beds. The department of orthopaedics is located at two sites, including the Hubin Campus and the Xiasha Campus, and has a total of 160 inpatient beds. All the patients will be recruited when

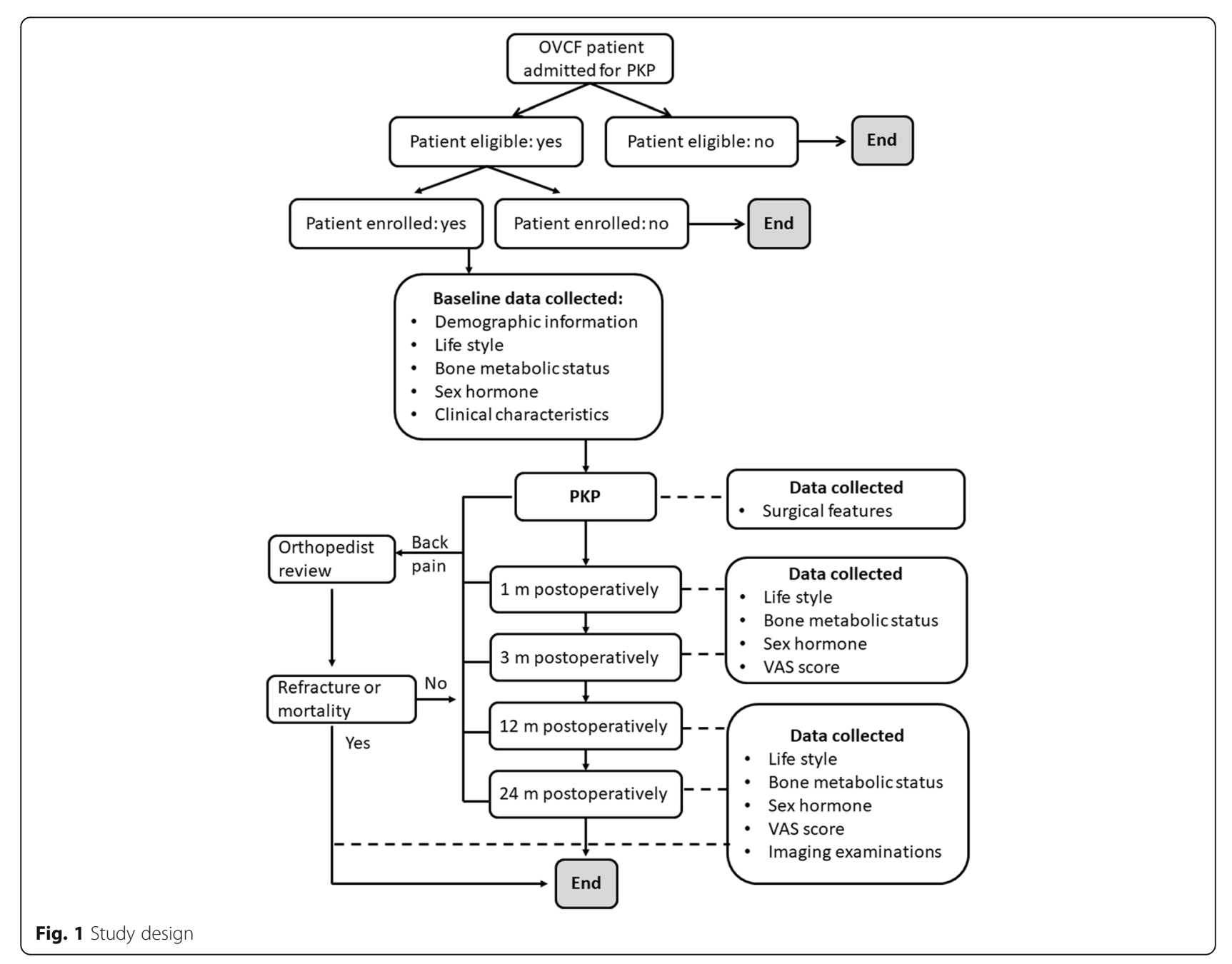


they are admitted to the hospital to undergo PKP to treat painful OVCFs.

\section{Inclusion and exclusion criteria}

The inclusion criteria are as follows: acute OVCFs of the thoracic, thoracolumbar, or lumbar spine with symptom duration of less than three weeks. An osteoporotic fracture is defined as a fracture that occurs spontaneously or secondary to mild trauma during daily activities. A VCF is diagnosed based on radiographs and computed tomography (CT) scans. Magnetic resonance imaging (MRI) examinations are carried out to confirm new OVCFs when patients are experiencing marked pain.

The exclusion criteria are as follows: A. previous vertebral fracture; B. previous major spinal surgery; C. complications involving malignant tumours; and D. fractures secondary to high-energy trauma, such as falls from high places.

\section{Sample size}

The sample size calculation was performed using Epi Info 7.2 software (www.cdc.gov/epiinfo/index.html) for the following risk factors: age and $E_{2}$ level. Based on our previous retrospective study, the ratio of patients < 80 years old and those $\geq 80$ years old is nearly $5: 1$, and the ratio of patients with $\mathrm{E}_{2}$ levels $\geq 50 \mathrm{pmol} / \mathrm{L}$ and those $<50 \mathrm{pmol} / \mathrm{L}$ is nearly $3: 1$ [24]. A two-sided test will be set with an $\alpha$ risk of 0.05 and a power of $80 \%$. Our previous retrospective study showed that the incidence of refracture was nearly $9 \%$ in the patients < 80 years old, $33 \%$ in patients $\geq 80$ years old, $2 \%$ in patients with $\mathrm{E}_{2}$ levels $\geq 50 \mathrm{pmol} / \mathrm{L}$, and $40 \%$ in patients with $\mathrm{E}_{2}$ levels $<50 \mathrm{pmol} / \mathrm{L}$. The calculated required sample size for the cohort is 164 patients to evaluate the risk factor of age and 50 patients to evaluate the risk factor of $E_{2}$ levels. Therefore, assuming a rate of loss to follow-up of $10 \%, 180$ participants will be included.

\section{Data collection}

As shown in Fig. 1, when a patient is enrolled in this study, the baseline data will be collected, including the demographic information, lifestyle, bone metabolic status, sex hormone levels, and clinical characteristics. The surgical features will be recorded during PKP. The assessment of lifestyle, bone metabolic status, sex hormone levels, SHBG levels, and clinical characteristics will be repeated at $1 \mathrm{~m}, 3 \mathrm{~m}, 12 \mathrm{~m}$, and $24 \mathrm{~m}$ postoperatively. Complications will be defined by the Post-Operative Morbidity Survey and recorded [34]. Those who develop aggravated back pain will be reviewed by an orthopaedist, and the information regarding lifestyle, bone metabolic status, sex hormone levels, SHBG levels, and imaging examinations will be collected if refracture or mortality is confirmed. All the data will be recorded by two researchers to ensure the accuracy of data.

\section{Anthropometric data}

The baseline data, including gender, age, weight, height, and BMI will be collected following participant recruitment (Table 1). Body weight and height will be measured twice with a height and weight scale (HW-900B, Kaiyuan Electronic Co., Zhengzhou, China) while the patients are wearing light clothes and are barefoot, and then the mean reading will be recorded. Body mass index (BMI) will be calculated as the body weight in kilograms divided by the square of the body height in metres.

\section{Lifestyle}

The patients' use of cigarettes will be assessed upon admission to the hospital and monitored during follow-up at $1 \mathrm{~m}, 3 \mathrm{~m}, 12 \mathrm{~m}$, and $24 \mathrm{~m}$ postoperatively.

To investigate smoking status, a questionnaire consisting of the following three questions will be used: (1) Have you ever smoked cigarettes regularly? (2) "Did you smoke prior to sustaining a vertebral compression fracture?" If yes, "How many cigarettes did you smoke each day on average?" If no, "How many months prior to sustaining a vertebral compression fracture did you stop smoking?" (3) "Have you smoked any cigarettes in the past seven days?" If yes, "How many cigarettes do you smoke each day on average?" If no, "When did you stopped smoking and why?" Then, the patients will be divided into never smokers, ex-smokers, current smokers, and heavy smokers.

\section{Bone mineral density}

BMD scores of the lower lumbar spine (L2-L4) and unilateral hip (total hip) will be measured using dual-energy X-ray absorptiometry (DXA) (Hologic, Waltham, MA, USA). Blinded professional trained technicians will perform the DXA scans. BMD will be expressed in $\mathrm{g} / \mathrm{cm}^{2}$ with $\mathrm{T}$-scores (standard deviation (SD) differing from the mean BMD of young women). Osteoporosis will be defined based on the region with the lowest BMD and classified according to the World Health Organization (WHO) classification. A T-score $>-1.0 \mathrm{SD}$ is defined as normal; a T-score ranging from $-1.0 \mathrm{SD}$ to $-2.5 \mathrm{SD}$ is defined as osteopenic; and a T-score $\leq-2.5 \mathrm{SD}$ is defined as osteoporotic.

\section{Bone metabolic markers}

Serum bone alkaline phosphatase (BAP) will be used as a marker of bone formation. BAP levels will be measured with an enzyme immunoassay (EIA) method (Sumitomo Biomedical, Osaka, Japan). 
Table 1 Timeline of data collection

\begin{tabular}{|c|c|c|c|c|c|c|c|c|}
\hline Data collection & & Pre & Op & $1 \mathrm{~m}$ & $3 \mathrm{~m}$ & $12 \mathrm{~m}$ & $24 \mathrm{~m}$ & OR \\
\hline \multirow[t]{5}{*}{ Demographic information } & Gender & $\sqrt{ }$ & & & & & & \\
\hline & Age & $\sqrt{ }$ & & & & & & \\
\hline & Height & $\sqrt{ }$ & & & & & & \\
\hline & Weight & $\sqrt{ }$ & & & & & & \\
\hline & BMl & $\sqrt{ }$ & & & & & & \\
\hline Life style & Tobacco consumption & $\sqrt{ }$ & & $\sqrt{ }$ & $\sqrt{ }$ & $\sqrt{ }$ & $\sqrt{ }$ & $\sqrt{ }$ \\
\hline \multirow[t]{3}{*}{ Bone metabolic status } & BMD & $\sqrt{ }$ & & $\sqrt{ }$ & $\sqrt{ }$ & $\sqrt{ }$ & $\sqrt{ }$ & $\sqrt{ }$ \\
\hline & Bone metabolic markers & $\sqrt{ }$ & & $\sqrt{ }$ & $\sqrt{ }$ & $\sqrt{ }$ & $\sqrt{ }$ & $\sqrt{ }$ \\
\hline & Anti-osteoporosis treatment & $\sqrt{ }$ & & $\sqrt{ }$ & $\sqrt{ }$ & $\sqrt{ }$ & $\sqrt{ }$ & $\sqrt{ }$ \\
\hline \multirow[t]{2}{*}{ Sex hormone } & Serum hormone concentrations & $\sqrt{ }$ & & $\sqrt{ }$ & $\sqrt{ }$ & $\sqrt{ }$ & $\sqrt{ }$ & $\sqrt{ }$ \\
\hline & SHBG & $\sqrt{ }$ & & $\sqrt{ }$ & $\sqrt{ }$ & $\sqrt{ }$ & $\sqrt{ }$ & $\sqrt{ }$ \\
\hline \multirow[t]{2}{*}{ Clinical characteristics } & Affected vertebra number & $\sqrt{ }$ & & & & & & \\
\hline & VAS score & $\sqrt{ }$ & & $\sqrt{ }$ & $\sqrt{ }$ & $\sqrt{ }$ & $\sqrt{ }$ & $\sqrt{ }$ \\
\hline \multirow[t]{4}{*}{ Surgical features } & Balloon volume & & $\sqrt{ }$ & & & & & \\
\hline & Cement volume & & $\sqrt{ }$ & & & & & \\
\hline & Vertebral height & $\sqrt{ }$ & $\sqrt{ }$ & & & $\sqrt{ }$ & $\sqrt{ }$ & $\sqrt{ }$ \\
\hline & Bone cement leakage & & $\sqrt{ }$ & & & & & \\
\hline
\end{tabular}

Pre: pre-operation. Op: operation. $1 \mathrm{~m}, 3 \mathrm{~m}, 12 \mathrm{~m}, 24 \mathrm{~m}$ : one month, three months, 12 months, 24 months postoperatively. OR Orthopedist review

Urinary $\mathrm{N}$-terminal cross-linking telopeptide of type I collagen (NTX) will be used as a marker of bone resorption. NTX levels will be examined with an enzyme-linked immunosorbent assay (ELISA) method (OSTEOMARK; Osteox International, Seattle, WA).

\section{Number of affected vertebra}

The number of affected vertebra will be assessed via preoperative imaging examinations, including radiographs, $\mathrm{CT}$, and MRI.

\section{Serum sex hormone concentration}

Serum levels of follicle-stimulating hormone (FSH), luteinizing hormone (LH), testosterone (TE), $\mathrm{E}_{2}$, and SHBG will be measured by electrochemiluminescence immunoassays (ECLIA) using a Roche Modular E170 immunoassay analyser (Roche Diagnostics, Indianapolis, IN, USA).

\section{VAS score}

The visual analogue scale (VAS) score will be assessed by a blinded trained nurse to estimate pain perception when the patient is admitted to the hospital and at $1 \mathrm{~m}$, $3 \mathrm{~m}, 12 \mathrm{~m}$, and $24 \mathrm{~m}$ after PKP [35]. The standard scale from 0 (no pain) to 10 (intolerable pain) will be adopted for pain analysis.

\section{Anti-osteoporotic treatment}

Zoledronic acid is an anti-osteoporosis agent that rapidly and markedly increases the BMD of vertebral bone. Injections of zoledronic acid (Aclasta, Novartis Pharma
Schweiz AG) will be recommended for patients who do not have contraindications for receiving zoledronic acid. If the patients agree to receive zoledronic acid treatment, $5 \mathrm{mg}$ of Aclasta (Novartis Pharma Schweiz AG) will be administered 3 days after PKP, and Vitamin D and calcium supplements will also be continued.

\section{PKP features}

Bipedicular kyphoplasty will be performed in this study. The balloon volume (BV), cement volume $(\mathrm{CV})$, recovery of vertebral height, and bone cement leakage will be evaluated as factors that influence the risk of vertebral refracture. 1) The balloon volume will be recorded by the surgeons during the operation. 2) The cement volume will be recorded by the surgeons during the operation. 3) The anterior height of the fractured vertebra will be immediately measured with the picture archiving and communication systems (PACS) imaging display software (Zhejiang Public Information Industry Co., China) when the patients are enrolled and at $12 \mathrm{~m}$ and $24 \mathrm{~m}$ after PKP. Recovery of vertebral height will be defined as the anterior height of the fractured vertebra/the average height of the two adjacent vertebrae. 4) Bone cement leakage will be evaluated using radiographic evaluation during operation.

\section{Analysis}

Quantitative variables will be summarized by the mean \pm $\mathrm{SD}$, and qualitative variables will be presented as counts and percentages. Continuous parameters of patients with 
and without vertebral refracture will be compared by analysis of variance (ANOVA), and binary parameters will be evaluated with logistic regression. A Pearson regression model will be used to analyse the correlation between the incidence of vertebral refracture and the covariates. Univariate and multivariate logistic regression analyses will be carried out to identify the independent predictors of vertebral refracture. A value of $P<0.05$ will be considered statistically significant. Statistical analyses will be carried out using the SPSS 19.0 statistics package (SPSS, Chicago, IL, USA).

\section{Amendment of the protocol}

A formal amendment to the protocol will be required before any modifications to the protocol are made. Any amendments will be approved by the Ethics Committee of the First Affiliated Hospital of Zhejiang Chinese Medicine University before implementation.

\section{Confidentiality}

Every participant will be coded by a unique number when they are included in this study. The unique number will be used to identify the participant throughout the study. The pairing between the participant and the identity number will be password protected.

\section{Ethics and dissemination}

This study has been approved by the Ethics Committee of the First Affiliated Hospital of Zhejiang Chinese Medicine University. This study has been registered online (http://www.chictr.org.cn/index.aspx) with the identifier ChiCTR-ROC-17011562 (registered on July 4th, 2017). The participants will be asked to sign an informed consent form before they are included in the study. The principles of the Declaration of Helsinki [36] and the WHO standards for observational studies [37] will be followed throughout the study.

A variety of methods will be adopted to maximize the visibility of the present study. First, we will try to publish this study protocol, which introduces the importance of evaluating the risk factors for vertebral refracture after PKP and describing the design. Second, to introduce the results, oral and poster presentations at national and international meetings will be encouraged to disseminate the findings. In addition, the study results will be published in a high-impact scientific journal to achieve maximum distribution.

We will deliver a deidentified dataset to the Clinical Trial Management Public Platform for release before December 2021.

\section{Discussion}

This study will explore the following risk factors for vertebral refracture after PKP: 1) general characteristics, lifestyle, bone metabolic status, and sex hormone levels to identify the patients tend to suffer from refracture after PKP and to provide sufficient consultation for these patients and 2) surgical features to identify the key points of PKP that may help to reduce the occurrence postoperative refracture. The results may provide new information about the OVCF patients suitable for PKP treatment.

The limitations of the present study are as follows. The current guidelines for the treatment of OVCF recommend a combination treatment method including anti-osteoporotic treatment regardless of the major treatment method used to reduce the incidence of refractures. Therefore, we used combination treatment with Aclasta to treat OVCF. However, when the results of this study are compared with other populations, the rates of adjacent level fractures as well as the risk factors for fractures after PKP may be different than those of other populations, such as populations not receiving any medical treatment or those receiving a different medication for osteoporosis.

\section{Abbreviations \\ ANOVA: Analysis of variance; BMD: Bone mineral density; BV: Balloon volume; CT: Computed tomography; CV: Cement volume; DXA: Dual-energy X-ray absorptiometry; $E_{2}$ : Oestradiol; ECLIA: Electrochemiluminescence immunoassays; ELISA: Enzyme-linked immunosorbent assay; FSH: Follicle-stimulating hormone; LH: Luteinizing hormone; MRI: Magnetic resonance imaging; NTX: N-terminal cross-linking telopeptide of type I collagen; OVCF: Osteoporotic vertebral compression fracture; PACS: Picture archiving and communication systems; PKP: Percutaneous kyphoplasty; RCT: Randomized controlled trial; SD: standard deviation; SHBG: Sex hormone binding globulin; TE: Testosterone; VAS: Visual analogue scale}

\section{Consent to publication}

Not applicable.

\section{Funding}

This study was sponsored by the following grants: Zhejiang Provincial Science and Technology Plan of Traditional Chinese Medicine (No. 2017ZQ013 to JMS); Medical and Health Science and Technology Plan of Zhejiang Province (No. 2017KY501 to JMS); Province Zhejiang Provincial Natural Science Foundation (No. LY17H040005 to CF); National Natural Science Foundation of China (No. 81701461 to CF).

\section{Availability of data and materials}

We will deliver a deidentified dataset to the Clinical Trial Management Public Platform for release before December 2021.

\section{Authors' contributions}

LF and JMS conceived the original study and developed the protocol together with CF. Statistical advice was provided by JC and YW. CF and YW wrote the manuscript. All authors read and approved the final manuscript.

\section{Ethics approval and consent to participate}

The study protocol has been approved by the Ethics Committee of the First Affiliated Hospital of Zhejiang Chinese Medicine University. The participants will be asked to sign an informed consent form before they are included in the study.

Competing interests

The authors declare that they have no competing interests. 


\section{Publisher's Note}

Springer Nature remains neutral with regard to jurisdictional claims in published maps and institutional affiliations.

\section{Author details \\ 'Department of Orthopaedics, The First Affiliated Hospital of Zhejiang Second Affiliated Hospital of Zhejiang University School of Medicine, Zhejiang 310006, Hangzhou, China. \\ Received: 12 September 2017 Accepted: 4 June 2018 Published online: 02 July 2018} Chinese Medicine University, 9 Ninth Avenue, Hangzhou Economic and Technological Development Zone, Zhejiang 310018, Hangzhou, China. ${ }^{2}$ The

\section{References}

1. Si L, Winzenberg TM, Jiang Q, Chen M, Palmer AJ. Projection of osteoporosis-related fractures and costs in China: 2010-2050. Osteoporos Int. 2015;26(7):1929-37.

2. Riggs BL, Melton $\amalg$ 3rd. The worldwide problem of osteoporosis: insights afforded by epidemiology. Bone. 1995;17(5 Suppl):505S-11S.

3. Kallmes DF, Comstock BA, Heagerty PJ, Turner JA, Wilson DJ, Diamond TH, Edwards R, Gray LA, Stout L, Owen S, et al. A randomized trial of vertebroplasty for osteoporotic spinal fractures. N Engl J Med. 2009;361(6):569-79.

4. Boonen S, Van Meirhaeghe J, Bastian L, Cummings SR, Ranstam J, Tillman JB, Eastell R, Talmadge K, Wardlaw D. Balloon kyphoplasty for the treatment of acute vertebral compression fractures: 2-year results from a randomized trial. J Bone Miner Res. 2011;26(7):1627-37.

5. Van Meirhaeghe J, Bastian L, Boonen S, Ranstam J, Tillman JB, Wardlaw D. A randomized trial of balloon kyphoplasty and nonsurgical management for treating acute vertebral compression fractures: vertebral body kyphosis correction and surgical parameters. Spine (Phila Pa 1976). 2013;38(12):971-83.

6. Clark W, Bird P, Gonski P, Diamond TH, Smerdely P, McNeil HP, Schlaphoff G, Bryant C, Barnes E, Gebski V. Safety and efficacy of vertebroplasty for acute painful osteoporotic fractures (VAPOUR): a multicentre, randomised, doubleblind, placebo-controlled trial. Lancet. 2016;388(10052):1408-16.

7. Yuan WH, Hsu HC, Lai KL. Vertebroplasty and balloon kyphoplasty versus conservative treatment for osteoporotic vertebral compression fractures: a meta-analysis. Medicine (Baltimore). 2016;95(31):e4491.

8. Trout AT, Kallmes DF, Kaufmann TJ. New fractures after vertebroplasty: adjacent fractures occur significantly sooner. AJNR Am J Neuroradiol. 2006;27(1):217-23.

9. Anderson PA, Froyshteter AB, Tontz WL Jr. Meta-analysis of vertebral augmentation compared with conservative treatment for osteoporotic spinal fractures. J Bone Miner Res. 2013;28(2):372-82.

10. Yi XLH, Tian FWY, Li CLH, Liu XLH. Recompression in new levels after percutaneous vertebroplasty and kyphoplasty compared with conservative treatment. Arch Orthop Trauma Surg. 2014;134(1):21-30.

11. Chen D, An ZQ, Song S, Tang JF, Qin H. Percutaneous vertebroplasty compared with conservative treatment in patients with chronic painful osteoporotic spinal fractures. J Clin Neurosci. 2014;21(3):473-7.

12. Wang B. A prospective randomized controlled study comparing the pain relief in patients with osteoporotic vertebral compression fractures with the use of vertebroplasty or facet blocking. European Spine JournalEur Spine J. 2016;25(11):3486-94.

13. Yang E-Z. Percutaneous Vertebroplasty versus conservative treatment in aged patients with acute osteoporotic vertebral compression fractures. Spine (Phila Pa 1976). 2016;41(8):654-60.

14. Farrokhi MRAE, Maghami Z. Randomized controlled trial of percutaneous vertebroplasty versus optimal medical management for the relief of pain and disability in acute osteoporotic vertebral compression fractures. J Neurosurg Spine. 2011;14(5):561-9.

15. Buchbinder RRPJPCSMB. A randomized trial of vertebroplasty for painful osteoporotic vertebral fractures. N Engl J Med. 2009;361(6):557-68.

16. Kroon FSM, Ebeling PRWJD, Osborne RHMPJ, Wriedt CHBR. Two-year results of a randomized placebo-controlled trial of vertebroplasty for acute osteoporotic vertebral fractures. J Bone Miner Res. 2014;29(6):1346-55.

17. Staples MBMPCJPRDR. New vertebral fractures after vertebroplasty: 2 -year results from a randomised controlled trial. Arch Osteoporos. 2015;10:2229.

18. Voormolen MH, Mali WP, Lohle PN, Fransen H, Lampmann LE, van der Graaf $Y$, Juttmann JR, Jansssens $X$, Verhaar HJ. Percutaneous vertebroplasty compared with optimal pain medication treatment: short-term clinical outcome of patients with subacute or chronic painful osteoporotic vertebral compression fractures. The VERTOS study. AJNR Am J Neuroradiol. 2007;28(3):555-60.
19. Klazen CA, Venmans A, de Vries J, van Rooij WJ, Jansen FH, Blonk MC, Lohle PN, Juttmann JR, Buskens E, van Everdingen KJ, et al. Percutaneous vertebroplasty is not a risk factor for new osteoporotic compression fractures: results from VERTOS II. AJNR Am J Neuroradiol. 2010;31(8):1447-50.

20. Blasco J, Martinez-Ferrer A, Macho J, San RL, Pomes J, Carrasco J, Monegal A, Guanabens N, Peris P. Effect of vertebroplasty on pain relief, quality of life, and the incidence of new vertebral fractures: a 12-month randomized follow-up, controlled trial. J Bone Miner Res. 2012;27(5):1159-66.

21. Rousing R, Hansen KL, Andersen MO, Jespersen SM, Thomsen K, Lauritsen $J M$. Twelve-months follow-up in forty-nine patients with acute/semiacute osteoporotic vertebral fractures treated conservatively or with percutaneous vertebroplasty: a clinical randomized study. Spine (Phila Pa 1976). 2010;35(5): 478-82.

22. Feng L, Shen JM, Feng C, Chen J, Wu Y. Comparison of radiofrequency kyphoplasty (RFK) and balloon kyphoplasty (BKP) in the treatment of vertebral compression fractures: a meta-analysis. Medicine (Baltimore). 2017; 96(25):e7150.

23. Takahara K, Kamimura M, Moriya H, Ashizawa R, Koike T, Hidai Y, Ikegami S, Nakamura Y, Kato H. Risk factors of adjacent vertebral collapse after percutaneous vertebroplasty for osteoporotic vertebral fracture in postmenopausal women. BMC Musculoskelet Disord. 2016;17:12.

24. Shen JM, Feng L, Chen J, Wu Y, Yu JN. Risk factors of new vertebral fracture PKP in patients with OVCF. Clinical Medcine of Zhejiang. 2017;19(5):806-8.

25. Martinez-Ferrer ABJ, Carrasco JLMJM, Roman LSLA, Monegal AGN, Peris P. Risk factors for the development of vertebral fractures after percutaneous vertebroplasty. J Bone Miner Res. 2013;28(8):1821-9.

26. Yang S. Risk factors and correlation of secondary adjacent vertebral compression fracture in percutaneous kyphoplasty. Int J Surg. 2016;36:13842

27. Lindsay R, Silverman SL, Cooper C, Hanley DA, Barton I, Broy SB, Licata A, Benhamou L, Geusens P, Flowers K, et al. Risk of new vertebral fracture in the year following a fracture. JAMA. 2001;285(3):320-3.

28. Vandenput L, Mellstrom D, Kindmark A, Johansson H, Lorentzon M, Leung J, Redlund-Johnell I, Rosengren BE, Karlsson MK, Wang YX, et al. High serum SHBG predicts incident vertebral fractures in elderly men. J Bone Miner Res. 2016;31(3):683-9.

29. Cawthon PM, Schousboe JT, Harrison SL, Ensrud KE, Black D, Cauley JA, Cummings SR, LeBlanc ES, Laughlin GA, Nielson CM, et al. Sex hormones, sex hormone binding globulin, and vertebral fractures in older men. Bone. 2016;84:271-8

30. Woods GN, Huang MH, Cawthon PM, Laughlin GA, Schousboe JT, McDaniels-Davidson C, Cauley JA, Orwoll E, Barrett-Connor E, Kado DM. SHBG, sex steroids, and kyphosis in older men: the MrOS study. J Bone Miner Res. 2016;31(12):2123-8.

31. Lin D, Hao J, Li L, Wang L, Zhang H, Zou W, Lian K. Effect of bone cement volume fraction on adjacent vertebral fractures after unilateral percutaneous Kyphoplasty. Clin Spine Surg. 2017;30(3):E270-270E275.

32. Jaramillo JD, Wilson C, Stinson DS, Lynch DA, Bowler RP, Lutz S, Bon JM, Arnold B, McDonald ML, Washko GR, et al. Reduced bone density and vertebral fractures in smokers. Men and COPD patients at increased risk. Ann Am Thorac Soc. 2015;12(5):648-56.

33. Weycker D, Edelsberg J, Barron R, Atwood M, Oster G, Crittenden DB, Grauer A. Predictors of near-term fracture in osteoporotic women aged $\geq 65$ years, based on data from the study of osteoporotic fractures. Osteoporos Int. 2017;28(9):2565-71.

34. Grocott MP, Browne JP, Van der Meulen J, Matejowsky C, Mutch M, Hamilton MA, Levett DZ, Emberton M, Haddad FS, Mythen MG. The postoperative morbidity survey was validated and used to describe morbidity after major surgery. J Clin Epidemiol. 2007;60(9):919-28.

35. Scott PJ, Huskisson EC. Measurement of functional capacity with visual analogue scales. Rheumatol Rehabil. 1977:16(4):257-9.

36. World Medical Association Declaration of Helsinki. ethical principles for medical research involving human subjects. JAMA. 2013;310(20):2191-4.

37. von EE, Altman DG, Egger M, Pocock SJ, Gotzsche PC, Vandenbroucke JP. The strengthening the reporting of observational studies in epidemiology (STROBE) statement: guidelines for reporting observational studies. Int J Surg. 2014;12(12):1495-9. 